\title{
EPR Evidence for Thermally Excited Triplet States in Exinite, Vitrinite and Inertinite Separated from Bituminous Coal
}

\author{
G.P. SŁowik, W. Wojtowicz And A.B. WiģCKowski \\ Institute of Physics, University of Zielona Góra \\ Prof. Z. Szafrana 4a, 65-516 Zielona Góra, Poland
}

\begin{abstract}
In this work we have made an electron spin resonance (EPR) study of macerals obtained from the lithotype clarain separated from Polish medium-rank coal ( 85.6 wt.\% C). For three macerals: exinite, vitrinite, and inertinite, the temperature dependence of intensity of EPR spectra in the temperature range of 100-373 K was investigated. The experimentally obtained EPR spectra of macerals were fitted by curves of the derivatives of the Gaussian and Lorentzian functions. The best fitting was obtained, when the experimental EPR spectra were assumed to be a superposition of three lines, for exinite and vitrinite - a broad Gaussian $(G)$, a broad Lorentzian $(L 1)$ and a narrow Lorentzian ( $L 3)$ line, but for inertinite of two lines - a narrow Lorentzian $(L 2)$ and a narrow Lorentzian (L3) line. The computer-assisted fitting has shown that each individual component line has similar values of resonance field, but different linewidths and amplitudes. The temperature dependence of line intensity $I$ of the broad Gaussian $(G)$ and narrow Lorentzian ( $L 2$ and $L 3$ ) lines fulfils the Curie law in the form $I=C / T$ or $I T=C$, whereas the broad Lorentzian $(L 1)$ line does not fulfil the Curie law. In the last case the temperature dependence of the Lorentzian $(L 1)$ component was fitted by the relation $I=C / T+B /[T(3+\exp (J / k T))]$ or $I T=C+B /[3+\exp (J / k T))]$, valid for thermally excited triplet states $(S=1)$. For exinite and vitrinite the curves presenting the temperature dependence of the product $I T$ versus temperature $T$ were resolved into two curves, one for paramagnetic centres in the doublet state $(S=1 / 2)$, and the other for paramagnetic centres in the thermally excited triplet state $(S=1)$.
\end{abstract}

PACS numbers: $61.66 . \mathrm{Hq}, 76.30 . \mathrm{Rn}, 87.64 . \mathrm{Hd}$

\section{Introduction}

The application of electron paramagnetic resonance (EPR) has indicated the existence of paramagnetic centres in thermally excited triplet states $(S=1)$ in 
bituminous coal [1]. In some papers [2] EPR spectra were analysed assuming the consistency of only one indivisible line indicating that all paramagnetic centres present in this substance fulfil the Curie law $(I=C / T)$. EPR spectra of macerals however are complex as demonstrated by numerical analysis of the lineshape $[3,4]$. Paramagnetic centres with thermally excited triplet states have been found to exist in exinite and vitrinite heated at $300^{\circ} \mathrm{C}$ and $650^{\circ} \mathrm{C}$ and in flame coal $[5,6]$. In some papers the authors suggest the existence of paramagnetic centres in quartet states $(S=3 / 2)$ (e.g. in fusinite and carbonized chars at $\left.720^{\circ} \mathrm{C}[7]\right)$. EPR saturation experiment have been interpreted using four groups of paramagnetic centres in macerals, making responsible them for four component lines in the form of Gaussian and Lorentzian functions (Gaussian $G$, Lorentzian $L 1$, Lorentzian $L 2$, Lorentzian L3) [8]. Thermally excited multiplet states apparently exist among paramagnetic centres responsible for the Lorentzian $L 1$ line found in exinite and vitrinite. It was ascertained that in heated inertinite paramagnetic centres with thermally excited triplet state do not exist [9].

\section{Experimental}

\subsection{Materials}

Studied macerals: exinite, vitrinite, and inertinite, were separated from clarain of Polish medium-rank coal $(85.6$ wt.\% C) characterized by very high purity (75 vol.\%, 90 vol.\%, 96 vol.\%, respectively). Exinite, vitrinite, and inertinite were separated by centrifugation of the demineralized clarain, one of lithotypes of coal, in toluene-carbon tetrachloride mixtures. To avoid interactions between molecules of atmospheric oxygen and paramagnetic centres present in exinite, vitrinite, and inertinite, the macerals samples were placed in thin-wall glass tubes and evacuated to $10^{-4}$ Torr and sealed. Macerals differ in physical and chemical properties, e.g. density and content of aromatic rings is growing in direction: exinite $<$ vitrinite $<$ inertinite.

\subsection{Measurements}

EPR measurements of macerals were performed using a spectrometer with microwave frequency $9.4 \mathrm{GHz}$ (X-band) and $100 \mathrm{kHz}$ magnetic field modulation. Microwave power incident on the cavity was approximately $0.7 \mathrm{~mW}$ in order to avoid signal saturation of studied macerals. Temperature was varried in the range of $173-373 \mathrm{~K}$ for exinite, $133-373 \mathrm{~K}$ for vitrinite, and 123-293 for inertinite. A ruby crystal was placed in the resonance cavity as a reference for line intensity measurements.

\subsection{Fitting process of EPR spectra}

The numerical analysis of EPR spectra for studied maceral samples has been performed after assuming that they are superpositions of the first derivative of Gaussian and Lorentzian functions. To establish the types and number of lines 
in EPR spectra of exinite, vitrinite, and inertinite, the least-square method was used.

\section{Results and discussion}

The best fitted experimental EPR spectra for exinite and vitrinite were obtained in the form of a sum of one Gaussian and two Lorentzians: a broad Lorentzian $L 1$ and narrow Lorentzian $L 3(G+L 1+L 3)$. For the inertinite sample the EPR spectra could be fitted by a superposition of two narrow Lorentzians $(L 2+L 3)$.

The various components of non-heated macerals (broad Gaussian $G$, broad Lorentzian $L 1$, narrow Lorentzian $L 2$, narrow Lorentzian $L 3$ ) are assigned for four different groups of paramagnetic centres existing in exinite, vitrinite, and inertinite. Paramagnetic centres responsible for each of the component lines belong to different aromatic structures [9]. In order to check the existence of thermally excited triplets in non-heated macerals, we have determined the values of amplitudes and linewidths of all components in the EPR spectra for exinite, vitrinite (Gaussian $G$, Lorentzian L1, Lorentzian L3) and inertinite (Lorentzian L2, Lorentzian L3). All these components of samples of exinite, vitrinite, and inertinite have very similar values of the resonance field $B_{\mathrm{r}}$.

The dependence of the integral intensity $I(I=C / T+B /[T(3+$ $\exp (J / k T))][10](J$ - singlet-triplet excitation energy) and the product of integral intensity-temperature $I T(I T=C+B /[3+\exp (J / k T)]$ versus temperature $T$ for all components are presented in Figs. 1 and 2. The behaviour of the intensity-temperature product $I T$ versus temperature $T$ was analysed. The paramagnetic doublet states $(S=1 / 2)$, which fulfil the Curie law in the form $I T=C$ in the coordinate system $I T$ versus $T$, give a straight line parallel to the abscissa. Graphs showing the theoretical dependence of intensity $I$ and the product integral intensity-temperature $I T$ versus temperature $T$, as well as reciprocal of temperature $1 / T$ are shown in Fig. 3. We have obtained straight lines on the graphs of the dependence of the product integral intensity-temperature $I T$ versus temperature $T$ for all components in the EPR spectra of exinite, vitrinite, and inertinite, except for the broad Lorentzian $L 1$ component in exinite $\left(\Delta B_{\mathrm{pp}}=0.67 \mathrm{mT}\right.$, room temperature, $20 \mathrm{~dB})$ and vitrinite $\left(\Delta B_{\mathrm{pp}}=0.69 \mathrm{mT}\right.$, room temperature, $\left.20 \mathrm{~dB}\right)$. The exinite and vitrinite samples apparently contain groups of paramagnetic thermally excited centres, which do not fulfil the Curie law. These centres exist as thermally excited triplet state $(S=1)$ together with additional overlapping paramagnetic centres in doublet ground state $(S=1 / 2)$ forming the broad Lorentzian $L 1$ line. For the Lorentzian $L 1$ component in the EPR spectra of exinite and vitrinite, the dependence of the product integral intensity-temperature $I T$ versus temperature $T$ is increasing with $T$. This line can be separated into two lines in the $I T$ versus $T$ plot: first line - originating from paramagnetic centres in their ground doublet state $(S=1 / 2)$, and second line - attributed to paramagnetic centres in 

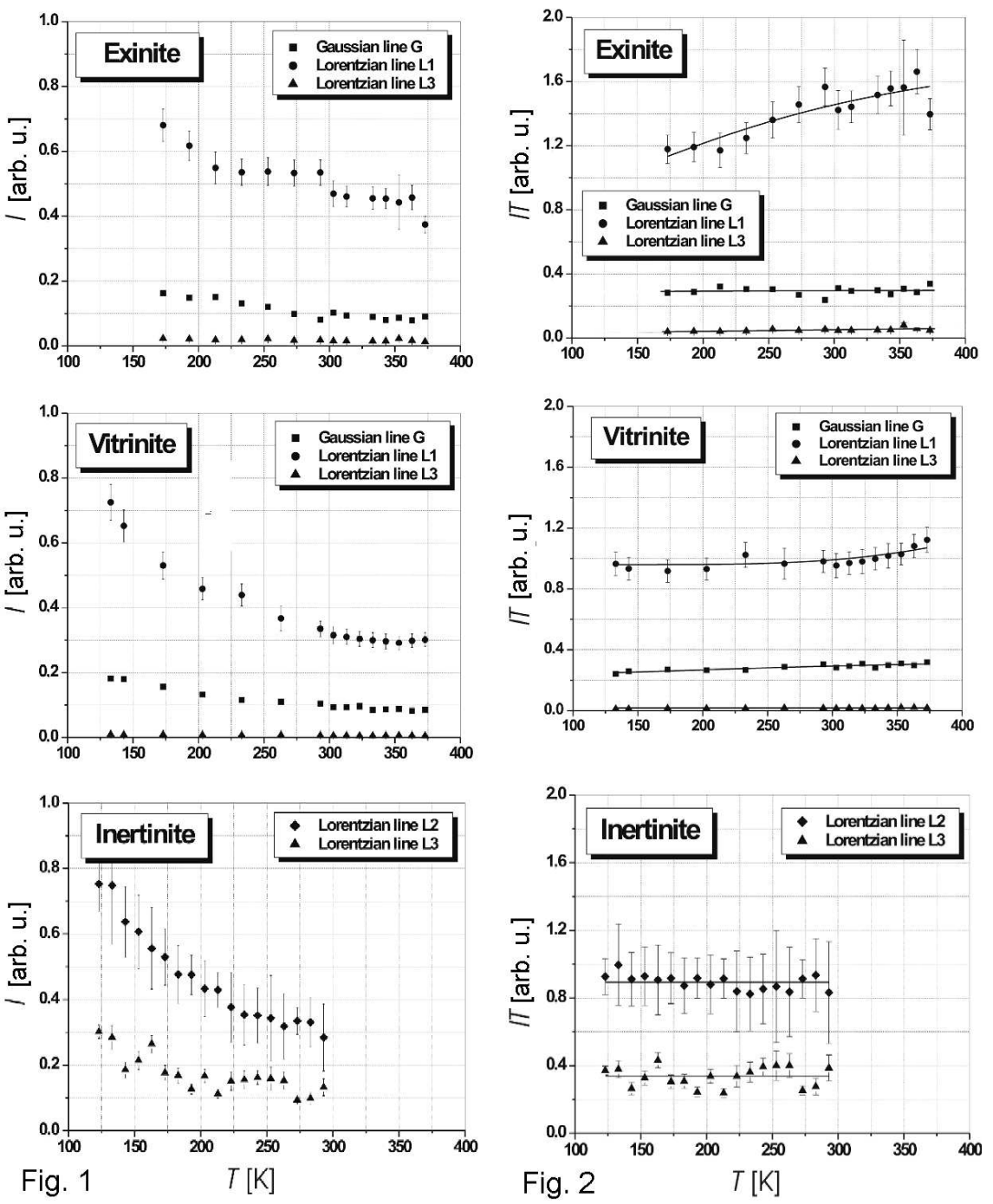

Fig. 1. Temperature dependence of intensities $I$ for the EPR components of exinite, vitrinite, and inertinite.

Fig. 2. Intensity-temperature product $I T$ versus temperature $T$ for the EPR components of exinite, vitrinite, and inertinite.

a thermally excited triplet state $(S=1)$, see Fig. 4 . The first component of the broad Lorentzian $L 1$ line is described by $(I T)_{1}=C$, and the second component by $(I T)_{2}=B /[3+\exp (J / k T)],(S=1)$. The values of energy separation $J / k$ of the thermally excited triplet states in the samples were estimated as $J / k=499 \mathrm{~K}$ and $J / k=1954 \mathrm{~K}$ for exinite and vitrinite, respectively. The percentage of paramagnetic centres in the thermally excited triplet state in exinite and vitrinite is 

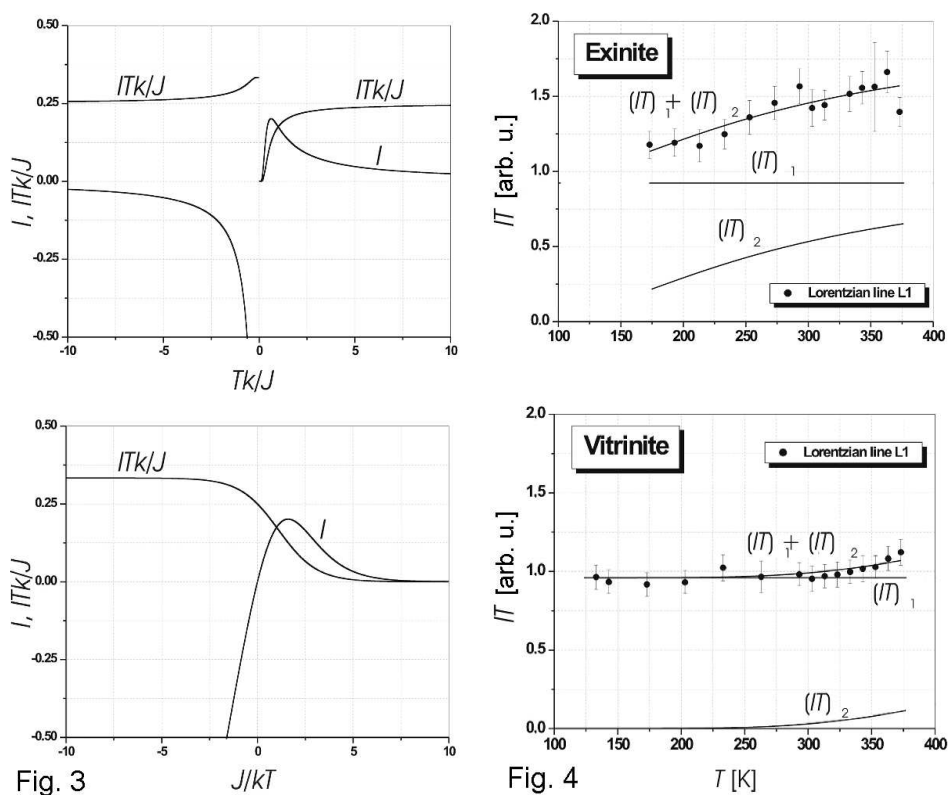

Fig. 3. Theoretical dependence (in dimensionless units) of intensity $I$ and intensitytemperature product $(I T k / J)$ versus temperature $T k / J$ (upper graph) and dependence of intensity $I$ and intensity-temperature product $(I T k / J)$ versus reciprocal of temperature $J / k T$ (lower graph) calculated with the formulae $I=1 /\{(T k / J)[3+\exp (J / k T)]\}$ and $I T k / J=1 /[3+\exp (J / k T)]$ valid for paramagnetic centres in thermally excited triplet state $(S=1)$. The value $T k / J$ in negative for separation energy $J<0$.

Fig. 4. Intensity-temperature product $(I T)$ versus temperature $T$ for Lorentzian $L 1$ line of exinite and vitrinite.

increasing with temperature and at room temperature is equal to about $13-18 \%$ of the total paramagnetic centres responsible for the broad Lorentzian $L 1$ line. The EPR study done in this work on inertinite showed also that for no component, neither the narrow Lorentzian $L 2\left(\Delta B_{\mathrm{pp}}=0.35 \mathrm{mT}\right.$, room temperature, $\left.20 \mathrm{~dB}\right)$, nor the narrow Lorentzian $L 3\left(\Delta B_{\mathrm{pp}}=0.13 \mathrm{mT}\right.$, room temperature, $\left.20 \mathrm{~dB}\right)$ lines, any paramagnetic centres in a thermally excited triplet state exist, and we can conclude that all components in the EPR spectrum of inertinite originate from paramagnetic centres in a ground doublet state. Similarly, the Gaussian component $G$ in exinite and vitrinite $\left(\Delta B_{\mathrm{pp}}=0.84 \mathrm{mT}\right.$, room temperature, $\left.20 \mathrm{~dB}\right)$ is generated only by paramagnetic centres in doublet ground state, too. For the existence of thermally excited triplet states in coal, donor-acceptor bonds between molecular and macromolecular phases can be responsible [1]. Other authors [7], make the spin clusters formed by several $\pi$ radicals coupled via a paramagnetic oxygen molecule, responsible for the appearance of multiplet states of paramagnetic centres in fusinite and carbohydrate chars. During the processes of thermal 
decomposition and reductive alkylation [6] of coals, the existence of thermally excited triplet states in macerals can be responsible for big changes of the amount and properties.

\section{Conclusions}

From this EPR study of non-heated macerals, exinite, vitrinite, and inertinite, obtained from clarain of Polish medium-rank coal, the following can be concluded:

1. There are three components in the EPR spectra of exinite and vitrinite (broad Gaussian $G$, broad Lorentzian $L 1$, narrow Lorentzian $L 3$ ) and two components for inertinite (narrow Lorentzian L2, narrow Lorentzian L3).

2. A numerical analysis showed that the broad Lorentzian $L 1$ line consists of centres in ground doublet state $(S=1 / 2)$, which obey the Curie law and also of centres in a thermally excited triplet state $(S=1)$. A small part (13-18\% in room temperature) of paramagnetic centres in examined macerals actually occupies the thermally excited triplet state $(S=1)$.

3. Non-heated inertinite samples do not contain paramagnetic centres in thermally excited triplet state.

We thank Professor Dr. Barbara Pilawa (Institute of Coal Chemistry, Polish Academy of Sciences, Gliwice) for the gift of maceral samples used in this study.

\section{References}

[1] S. Duber, A.B. Więckowski, Fuel 63, 1474 (1984).

[2] K.S. Rothenberger, R.F. Sprecher, S.M. Castellano, H.L. Retcofsky, in: Magnetic Resonance of Carbonaceous Solids, Eds. R.E. Botto, Y. Sanada, Advances in Chemistry Series, Vol. 229, American Chemical Society, Washington (DC) 1993, p. 581.

[3] R.B. Clarkson, Wei Wang, D.R. Brown, H.C. Crookham, R.L. Belford, in: Magnetic Resonance of Carbonaceous Solids, Eds. R.E. Botto, Y. Sanada, Advances in Chemistry Series, Vol. 229, American Chemical Society, Washington 1993, p. 507.

[4] B. Pilawa, A.B. Więckowski, B. Trzebicka, Radiat. Phys. Chem. 45, 899 (1995).

[5] A.B. Więckowski, B. Pilawa, L. Świątkowska, W. Wojtowicz, G.P. Słowik, M. Lewandowski, J. Magn. Reson. 145, 62 (2000).

[6] A.B. Więckowski, B. Pilawa, W. Wojtowicz, G.P. Słowik, H. Wachowska, Fuel 80, 451 (2001).

[7] T.I. Smirnowa, A.I. Smirnow, R.B. Clarkson, R.L. Belford, J. Phys. Chem. 98, 2464 (1994).

[8] G.P. Słowik, A.B. Więckowski, Appl. Magn. Reson. 24, 437 (2003).

[9] S. Schlick, M. Narayana, L. Kevan, Fuel 62, 1250 (1983).

[10] W.E. Hatfield, in: Theory and Applications of Molecular Paramagnetism, Eds. E.A. Boudreaux, L.N. Mulay, Wiley, New York 1976, ch. 7, p. 349. 